Finally the salt issue. Still a matter of controversy after so many years of research, discussions and official recommendations. Basic aspects are dealt with by Cesar Romero introducing us to a new regulatory entity called "natriuretic-ureotelic regulation". Another topic he discusses is the role of chloride vs other anions as partners of sodium. In experiments on genetically hypertensive rats, $\mathrm{F}$. Luft and al (Clin Sci 1988;74(6):577-85) have already demonstrated many years ago that drinking a sodium chloride- but not sodium bicarbonate solution can increase blood pressure. New basic data discussed at the Glasgow meeting may shed some light on underlying mechanisms. In addition, exciting new results point to an association of high salt intake and cognitive impairment involving the Tau protein which has long been recognized to be involved in Alzheimer's disease. The last paper of this section by Neusa Jessen deals in a very balanced way with several clinical aspects of the salt story discussed at the Glasgow meeting. New data by Franz Messerli and colleagues pointing to a lack of an association between salt intake and premature death are discussed in the light of the large body of evidence accusing a high salt diet to be an important risk factor for hypertension and cardiovascular disease. Recent data suggest that some biomarkers may be able to better distinguish between salt-sensitive and salt-resistant individuals, and there was even a recommendation that salt food "fortification" with nitrate-rich vegetable extracts may be a better solution to the problem than reducing salt in the diet.

\title{
Immunity and Inflammation in Hypertension - A Long Way To Go
}

\author{
FRANCISCO J RIOS \\ Institute of Cardiovascular and Medical Sciences, BHF Glasgow Cardiovascular Research \\ Centre, University of Glasgow, \\ Glasgow, UK.
}

\section{DOI:10.30824/2106-3}

The immune system is often defined as a complex network to protect the body against the invasion of microorganisms. In fact, what we most know about the immune response is derived from studies observing the immune response over a challenge mediated by pathogens or non-self-molecules. This traditional concept is not too far from the truth; however, it is a rather simplistic way to define such a complex system. According to Prof Cohen "The immune system has earned a reputation, justly, for its role as protector of the body against foreign invaders. However, the immune system is not only a department of defence, it also functions as a department of internal welfare" ${ }^{\prime \prime}$. Following this line of thinking, we should dare to consider that the main function of the immune system is to control the body homeostasis and, in only extreme cases this system faces an infection. This complex network is achieved by a group of circulating and tissue resident cells that patrol vessels and tissues to scavenge cells debris or DAMPS (Danger Associated Molecular
Patterns) to avoid unnecessary activation. In stress conditions, the immune system acts to eliminate the stressor agent and ultimately to return the system to the homeostatic state ${ }^{2}$. However, as the old proverb says: "the road to hell is paved with good intentions"; Situations where stressor agents are constantly produced will lead to uncontrolled immune/inflammatory activation, and a response that was primarily induced to eliminate the stressor agent, now can potentially lead to chronic inflammatory diseases and tissue damage.

Given the impact of the immune system in cardiovascular diseases and that patients with high blood pressure exhibit increased plasma inflammatory mediators and immune cells infiltration in the cardiovascular and renal tissues, it comes as no surprise that biomedical research is focused on applying these observations to hypertension research, and this has been under intense investigation in the past 15 years ${ }^{3,4,5}$. 
At Hypertension 2021, we could witness the presentation of very elegant and high-quality research discussing the importance of the inflammatory and immune response in the pathogenesis of hypertension. Studies were very diverse, as observed in the workshop "Immunity and inflammation in hypertension" chaired by Prof Guzik and Prof Schiffrin. Prof Lembo presented exciting data about how the activation of the CNS (Central Nervous System) can contribute to hypertension development by stimulating spleens to produce and activate the PIGF (Placenta growth factor) - Nrp-1 (neuropilin-1) axis, which induce the activation/maturation of dendritic cells and consequently activate CD8+ T lymphocytes to induce target organ damage. Important innate immunity mechanisms in hypertension development were also well discussed by Prof Drummond, who focused his presentation on inflammasome activation. According to his data, important adaptors of the inflammasome pathway, such as NLRP-3 (NOD-, LRR- and pyrin domain-containing protein 3), ASC (adaptor molecule apoptosis-associated speck-like protein containing a CARD) and IL-18 (interleukin-18), contribute to the major inflammatory response and organ damage in experimental models of hypertension. These two presentations highlight the importance of the two arms of the immune response in the hypertension research: the adaptive immunity, (mediated by lymphocytes) and innate immunity (mediated by monocytes/ macrophages, dendritic cells). Of importance, vascular cells also participate actively in the innate immune response, by inflammasome activation and production of inflammatory mediators. An interesting clinical study presented by Dr Crouch in the in the Award Session of the New Investigator Committee showed a negative correlation of urinary potassium concentration and inflammatory mediators observed in hypertensive patients with low salt consumption. Suggesting that potassium may have an anti-inflammatory effect but only in white population and under low salt intake. This clinical study demonstrates how complex are the mechanisms of the inflammation/immune activation and suggests that more causative and temporal studies are necessary to draw a clear understand of the immune system in the pathogenesis of hypertension.
The importance of the inflammation in the CNS was also addressed by Dr Shinohara in the $19^{\text {th }}$ International SHR Symposium, who showed that macrophages depletion in SHRSP (Spontaneously Hypertensive Stroke Prone Rat) reduces the expression of cyclooxygenase-2 in the brain, neuron activity, and blood pressure. Interestingly, in his data, macrophage depletion did not change the plasma levels of IL-1 $\beta$, thus suggesting the participation of other cells than macrophages in the inflammatory response observed in the SHRSP. Of note, IL-1 $\beta$ is a product of the inflammasome activation, which is also observed in vascular cells. Whether these effects have impact on cognition still needs elucidation. But it certainly opens doors for new research in aging and vascular dementia.

Hypertension is a multifactorial disease, that involves different pressor mediators, oxidative stress, genetic predisposition, and lifestyle. The immune system is educated according to the challenges received over the years, and if individuals with predisposition to hypertension have more pressor mediators and oxidative stress, the low-grade inflammation will be present during life. Therefore, it is expected that the inflammation and immune response observed in these different scenarios are different, at least qualitatively. Current research about the immune system in hypertension models shows the importance of activation of subpopulations of macrophages, dendritic cells presenting modified endogenous molecules, activation of CD4+ and CD8+ T lymphocytes, including subpopulations of CD4+ $T$ cells, such as regulatory $T$, and more recently gamma-delta $T$ cells in several hypertensive models ${ }^{5,4}$. The activation of granulocytes has also been observed, since NET (Neutrophil Extracellular Traps) formation by activated neutrophils are important mediators of vascular injury ${ }^{6,7}$. Therefore, it is important to determine whether these different arms of the inflammation and immune activation system play an important role according to time and model of hypertension development and organ damage. All these studies are very exciting and bring new paradigms and concepts to the research in hypertension. 


\section{References:}

1. Cohen IR. Chapter 1 - Introduction. In: Cohen IR, editor. Tending Adam's Garden. London: Academic Press; 2000. p. 3-6.

2.Chovatiya R, Medzhitov R. Stress, inflammation, and defense of homeostasis. Mol Cell. 2014;54(2):281-8. DOI: 10.1016/j.molcel.2014.03.030

3.Guzik TJ, Hoch NE, Brown KA, McCann LA, Rahman A, Dikalov $S$, et al. Role of the T cell in the genesis of angiotensin II induced hypertension and vascular dysfunction. J Exp Med. 2007;204(10):2449-60. DOl: 10.1084/jem.20070657

4.Caillon A, Paradis P, Schiffrin EL. Role of immune cells in hypertension. Br J Pharmacol. 2019;176(12):181828. DOI: 10.1111/bph.14427
5. Harrison DG, Coffman TM, Wilcox CS. Pathophysiology of Hypertension: The Mosaic Theory and Beyond. Circ Res. 2021;128(7):847-63. DOI: 10.1161/CIRCRESAHA.121.318082

6. McCarthy CG, Saha P, Golonka RM, Wenceslau CF, Joe B, Vijay-Kumar M. Innate Immune Cells and Hypertension: Neutrophils and Neutrophil Extracellular Traps (NETs). Compr Physiol. 2021;11(1):1575-89. DOI: 10.1002/cphy.c200020

7. Molinaro R, Yu M, Sausen G, Bichsel CA, Corbo C, Folco EJ, et al. Targeted delivery of Protein Arginine Deiminase-4 inhibitors to limit arterial intimal NETosis and preserve endothelial integrity. Cardiovasc Res. 2021. DOI: 10.1093/cvr/cvab074

\title{
COVID19 and Hypertension: Are We Facing a Syndemic?
}

\author{
NICOLAS F. RENNA, \\ Department of Pathology, Hospital Español de Mendoza, National University of \\ Cuyo, IMBECU-CONICET, \\ Mendoza, Argentina.
}

\section{DOI:10.30824/2106-4}

The term "syndemic" refers to synergistic health problems that affect the health of a population in its social and economic context. Coronavirus disease 2019 (COVID-19), indiced severe, acute respiratory syndrome Coronavirus 2 (SARSCoV-2), has become a global pandemic that is responsible for millions of deaths worldwide. High blood pressure is an important risk factor for cardiovascular disease and causes 7.5 million deaths per year (12.8\% of all deaths annually). The global burden of disease study suggests that systolic blood pressure is accountable for the highest proportion of premature death, with 212 million years lost ${ }^{1}$.

Early benign case series did not indicate an excess of hypertension in people admitted to hospital with COVID-192. Some later data showed a higher case fatality in patients with hypertension although not related for age ${ }^{3}$. New evidence from medical records in England, suggests that hypertension is not associated with hospital mortality from COVID -19 , hazard ratio (HR) 0.95, (95\% Cl 0.89-1.01). In sensitivity analyses, self-diagnosed hypertension

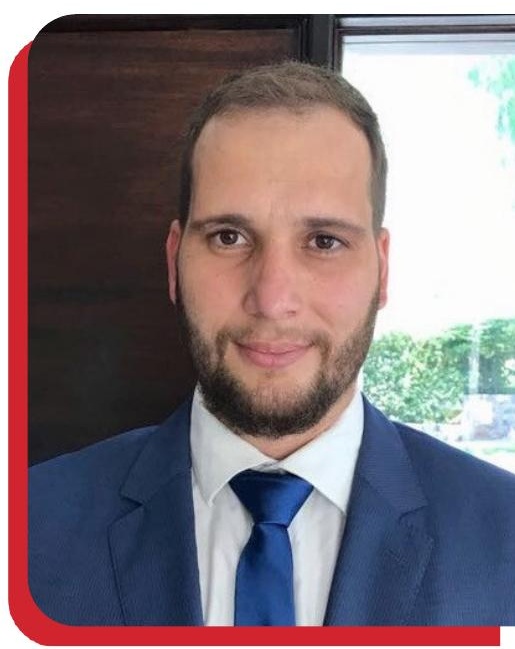

was associated with a slightly higher risk (HR 1.07, $95 \% \mathrm{Cl} 1.00-1.15)^{4}$.

Although some studies have concluded that hypertension could be a clinical predictor of severity ${ }^{5}$, the mechanism by which hypertension leads to an increased risk of COVID-19 is undoubtedly complex and may well be related to the underlying comorbidity. The prognosis for people with hypertension is markedly worse when COVID-19 infection is complicated by myocardial injury and in the presence of cardiovascular disease ${ }^{6}$. Target organ damage and cardiovascular events associated with poor blood pressure control increase with age. Therefore, it seems plausible that they may explain the observed associations between age, hypertension, and severity of COVID19 infection?.

\section{Are antihypertensive medications a serious risk of COVID-19 disease?}

From the beginning of the pandemic, many patients wondered whether they should 